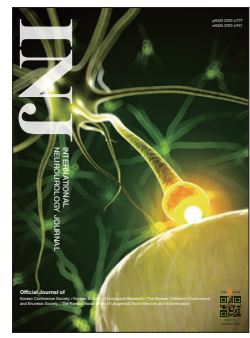

\title{
Robotic Sacrocolpopexy for Treatment of Apical Compartment Prolapse
}

\author{
Kwang Jin $\mathrm{Ko}^{1}$, Kyu-Sung Lee ${ }^{2,3}$ \\ ${ }^{1}$ Department of Urology, Kangnam Sacred Heart Hospital, Hallym University College of Medicine, Seoul, Korea \\ ${ }^{2}$ Department of Urology, Samsung Medical Center, Sungkyunkwan University School of Medicine, Seoul, Korea \\ ${ }^{3}$ Department of Medical Device Management and Research, SAIHST, Sungkyunkwan University, Seoul, Korea
}

\begin{abstract}
Abdominal sacrocolpopexy is the gold-standard treatment for apical compartment prolapse, as it is more effective and durable than the transvaginal approach. In the current era of minimally invasive surgery, laparoscopic sacrocolpopexy techniques have been described, but have not gained popularity due to their complexity and steep learning curves. To overcome this problem, robotic sacrocolpopexy was introduced, and has shown equivalent outcomes and safety compared to open and laparoscopic sacrocolpopexy based on findings that have been accumulated over 15 years.
\end{abstract}

Keywords: Pelvic organ prolapse; Robotics; Sacrocolpopexy

- Conflict of Interest: The authors have no potential conflicts of interest relevant to this article.

\section{INTRODUCTION}

The incidence of pelvic organ prolapse (POP) has been gradually increasing with the global increase in the aging population, and the lifetime risk of symptomatic vaginal prolapse requiring surgical treatment is estimated to be $12.6 \%[1,2]$. Although anterior vaginal prolapse is the most common type of prolapse, apical prolapse is also highly prevalent. It has been estimated that 1 in 9 women undergoes hysterectomy during her lifetime, and surgical treatment is required in $10 \%$ of them due to symptomatic vaginal prolapse [3]. Moreover, apical support defects after hysterectomy are a risk factor for POP and increase the possibility of vault prolapse.

abdominal sacrocolpopexy (ASC) remains the gold-standard procedure for apical compartment prolapse, as it offers superior outcomes for a variety of vaginal procedures with few compli- cations [3]. In addition to its high success rate and durable results, sacrocolpopexy can maintain the normal axis of the vagina and maximal vaginal length by fixing the vaginal apex on the anterior surface of the sacrum. Sacrocolpopexy is traditionally performed via laparotomy; however, minimally invasive approaches have been developed to overcome concerns about the increased risk of morbidity associated with open surgery, in addition to long surgery times and lengthy hospital stays. While reports have indicated that laparoscopic sacrocolpopexy (LSC) has the advantages of a shorter hospital stay and less blood loss, surgical time is not significantly shorter, and this technique has a longer learning curve than traditional sacrocolpopexy [4].

To address these issues, robotic sacrocolpopexy (RSC) has been explored. After Di Marco et al. [5] published the first case series involving 5 patients who underwent RSC in 2004, many more reports have been published. The ultimate advantages of

Corresponding author: Kyu-Sung Lee (DD https://orcid.org/0000-0003-0891-2488 Department of Urology, Samsung Medical Center, Sungkyunkwan University, School of Medicine, 81 Irwon-ro, Gangnam-gu, Seoul 06351, Korea

E-mail: ksleedr@skku.edu

Submitted: February 10, 2020 / Accepted after revision: April 24, 2020 


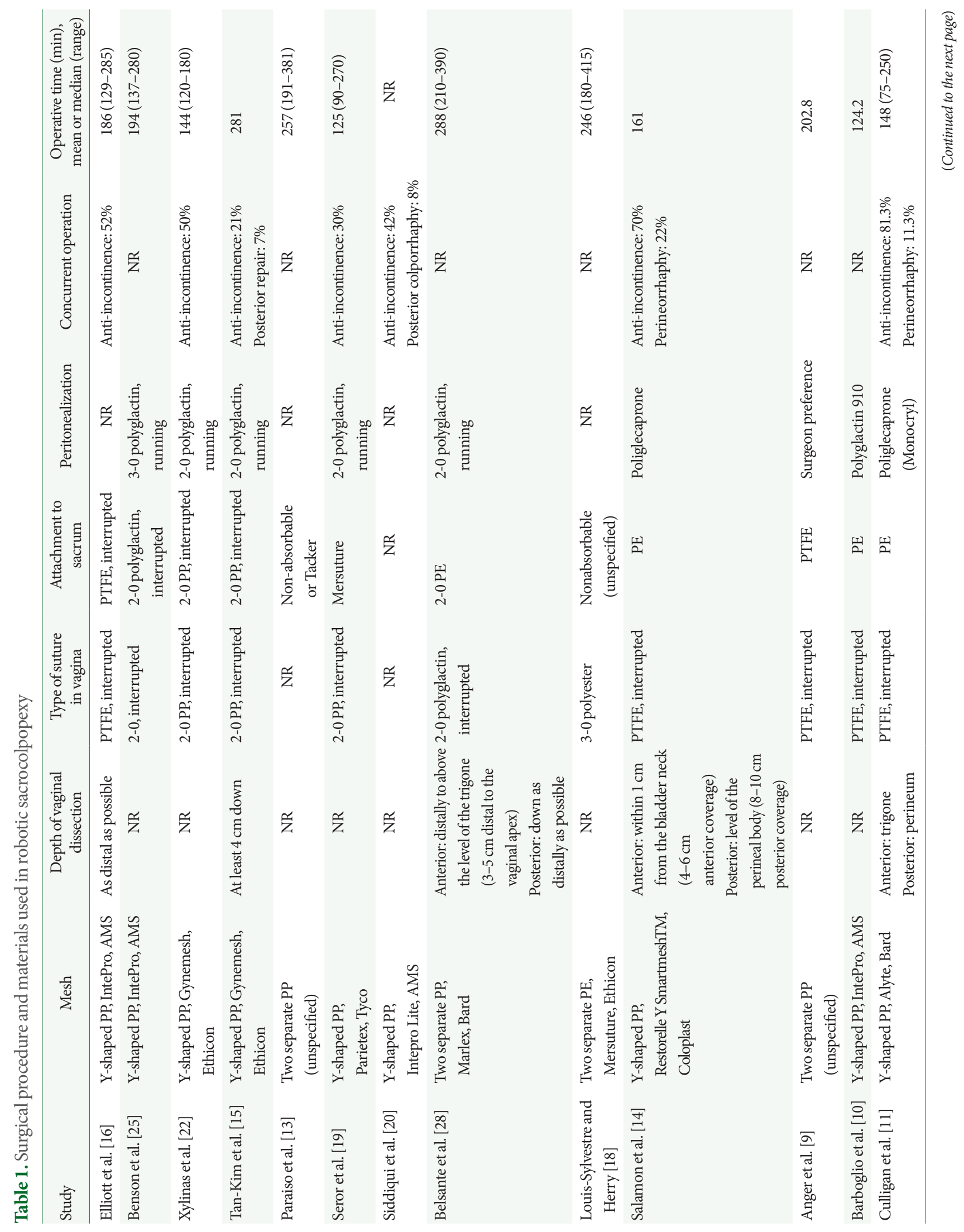




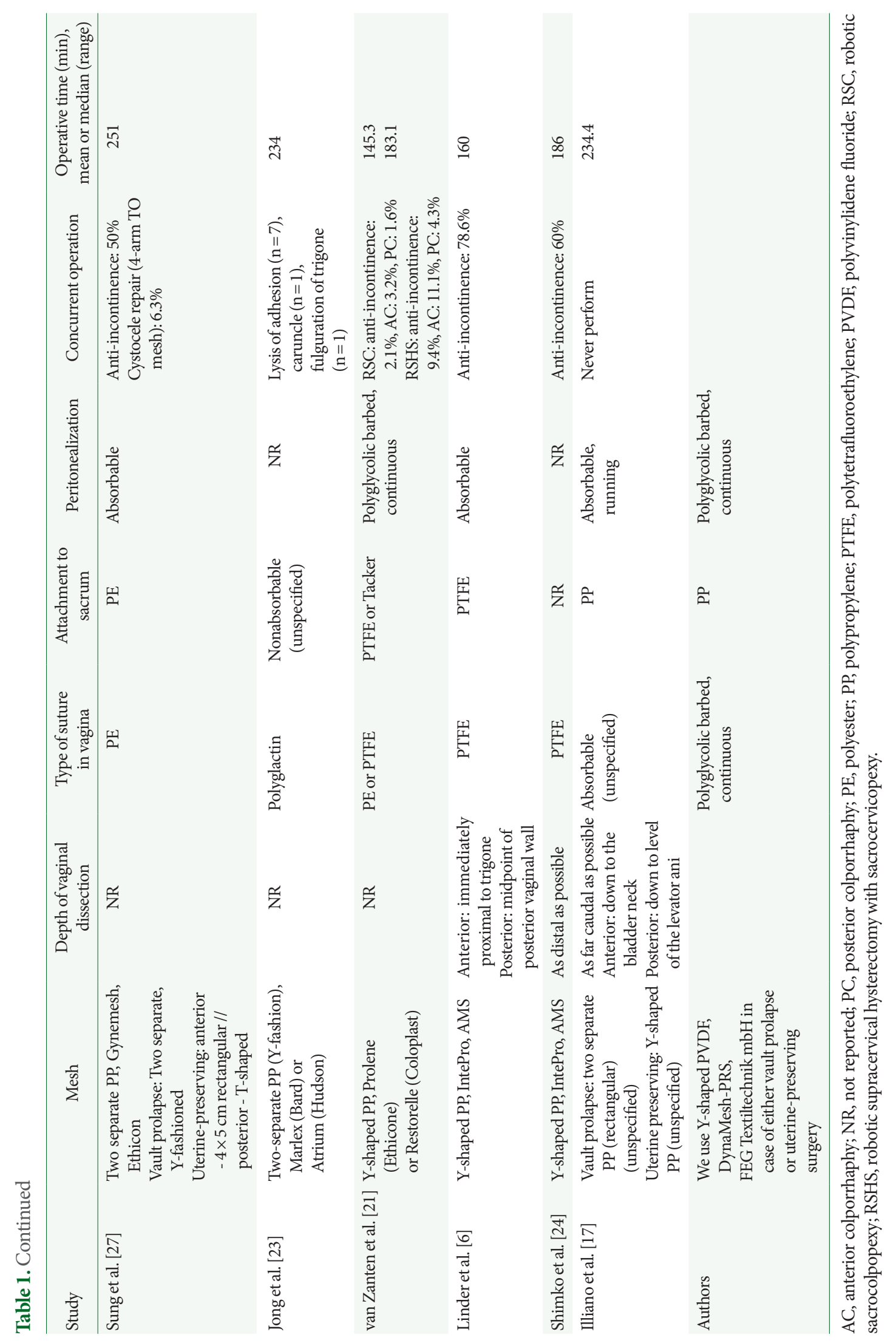


RSC are the 3-dimensional view afforded by the use of robotic instruments, the increased degree of freedom in movement, elaborate suturing ability, and easy knot-tying. These advantages of robotic surgery can overcome the technical limitations and steep learning curve associated with LSC. In this review, we discuss the efficacy and safety of RSC, as well as the latest trends in this field.

\section{SURGICAL TECHNIQUE}

RSC consists of 3 main steps: vaginal dissection, presacral peritoneal dissection, and mesh fixation. However, as shown in Table 1 , the type of mesh, suture material, and depth of vaginal dissection vary among studies.

The shape of the mesh used (Y-shaped mesh, 2 separate meshes, T-shaped mesh, or racket-shaped mesh) varies depending on the surgeon, and the depth dissected is often not mentioned (Ta- ble 1). Thus far, there are no clear rules as to the extent of anterior dissection that should be performed. A commonly accepted rule is to dissect as distally as possible to prevent recurrence, but not below the trigone. We apply this rule to dissect the anterior vaginal wall distally to just above the level of the trigone $(3-5 \mathrm{~cm}$ distal to the vaginal apex), and the posterior wall to the midpoint (Fig. 1). We use prefashioned Y-shaped DynaMesh, which is a polyvinylidene fluoride monofilament material, for sacrocolpopexy or hysteropexy (for uterine preservation). In patients who have previously undergone hysterectomy, the anterior and posterior vaginal walls are dissected under guidance of a vaginal manipulator, and then the distal 2-arm of mesh is fixed to the anterior and posterior vaginal walls (Fig. 2). When the uterus has been preserved, the mesh is transferred through the broad ligament with a proximal arm on the right side (Fig. 3).

Currently, the consensus is to affix the sacral arm of the mesh
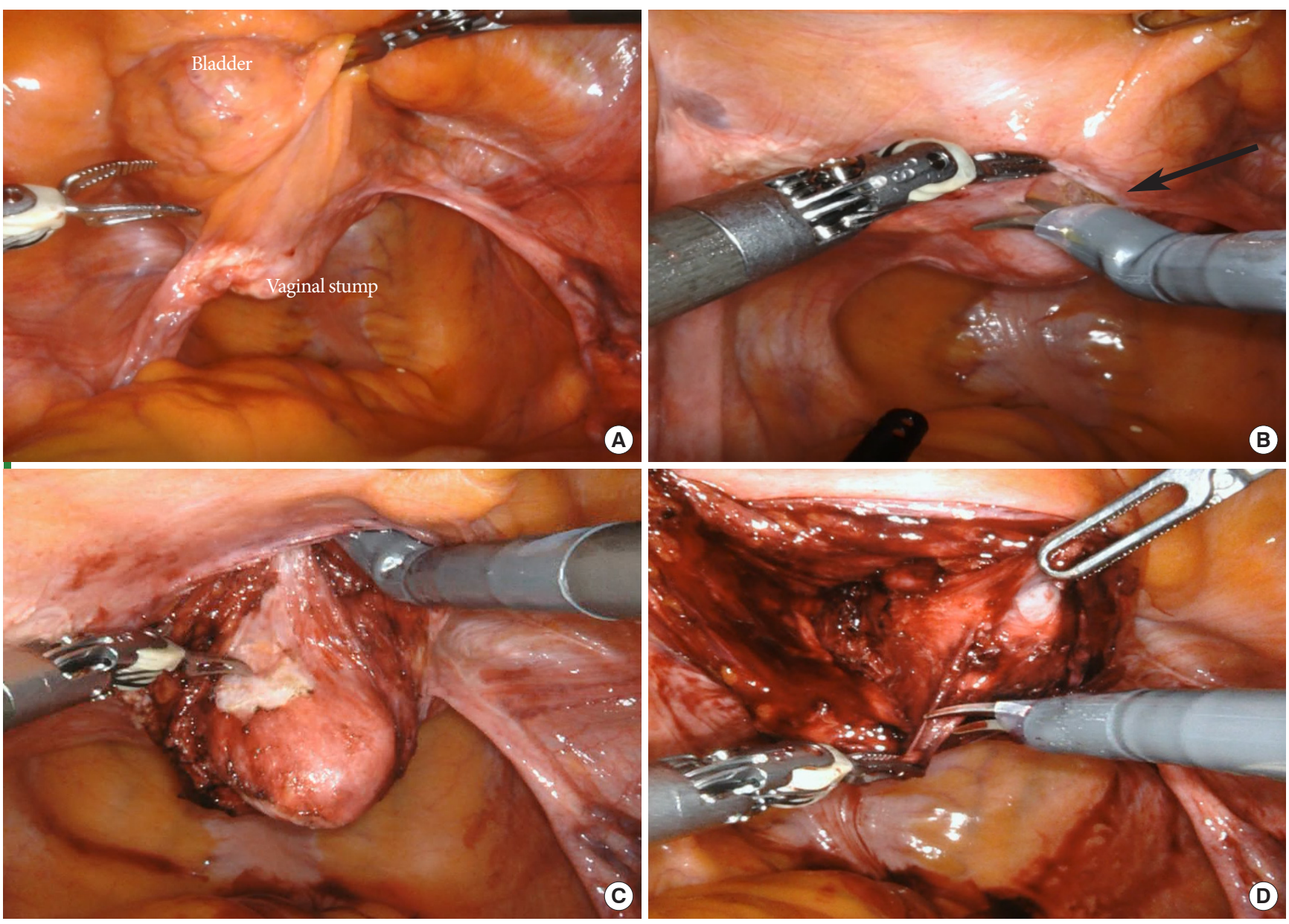

Fig. 1. Vaginal wall dissection (posthysterectomy vaginal vault prolapse). (A) Surgical anatomy of the vaginal apex and bladder, (B, C) anterior vaginal wall dissection (black arrow: vesicovaginal junction), and (D) posterior vaginal wall dissection. 
to the most superior point of the anterior surface of S1. However, although the bleeding risk is lower at the S1 level than at other levels, the promontory is not anatomically familiar, and there is a risk of bleeding due to the close proximity of nerves to the surrounding blood vessels. Therefore, caution is required when performing dissection.

A nonabsorbable suture is used in traditional open sacrocolpopexy to prevent detachment of the mesh from the vagina and sacral promontory and to decrease the risk of mesh exposure and suture erosion. In a series of RSCs with a median of 33 months of follow-up, the use of absorbable sutures for both vaginal and sacral mesh attachment was effective; the 3-year rate of survival without repeat prolapse surgery was 93\% [6]. Tan-Kim et al. [7] introduced a technique that fixes mesh to the vaginal wall with a barbed absorbable suture and showed that the nonbarbed suture group had significantly longer operative times than the barbed suture group (42 minutes vs. 29 minutes, $\mathrm{P}<0.001$ ) without any significant difference in anatomic failure between groups at 12 months. Currently, we use a barbed, delayed-absorbable suture (V-Loc 180; Covidien, Walpole, MA, USA) to fix the mesh to the vaginal wall in a continuous manner (Figs. 2, 3). In the next step, with the vagina restored to its normal position, the proximal arm of the mesh is fixed to the promontory without excessive tension (Fig. 4). Finally, the peritoneum is repaired using a barbed, delayed-absorbable suture.

\section{SURGICAL OUTCOMES}

\section{Outcomes of RSC and Heterogeneity of Surgical Methods}

With regard to the efficacy of RSC, the objective cure rate was
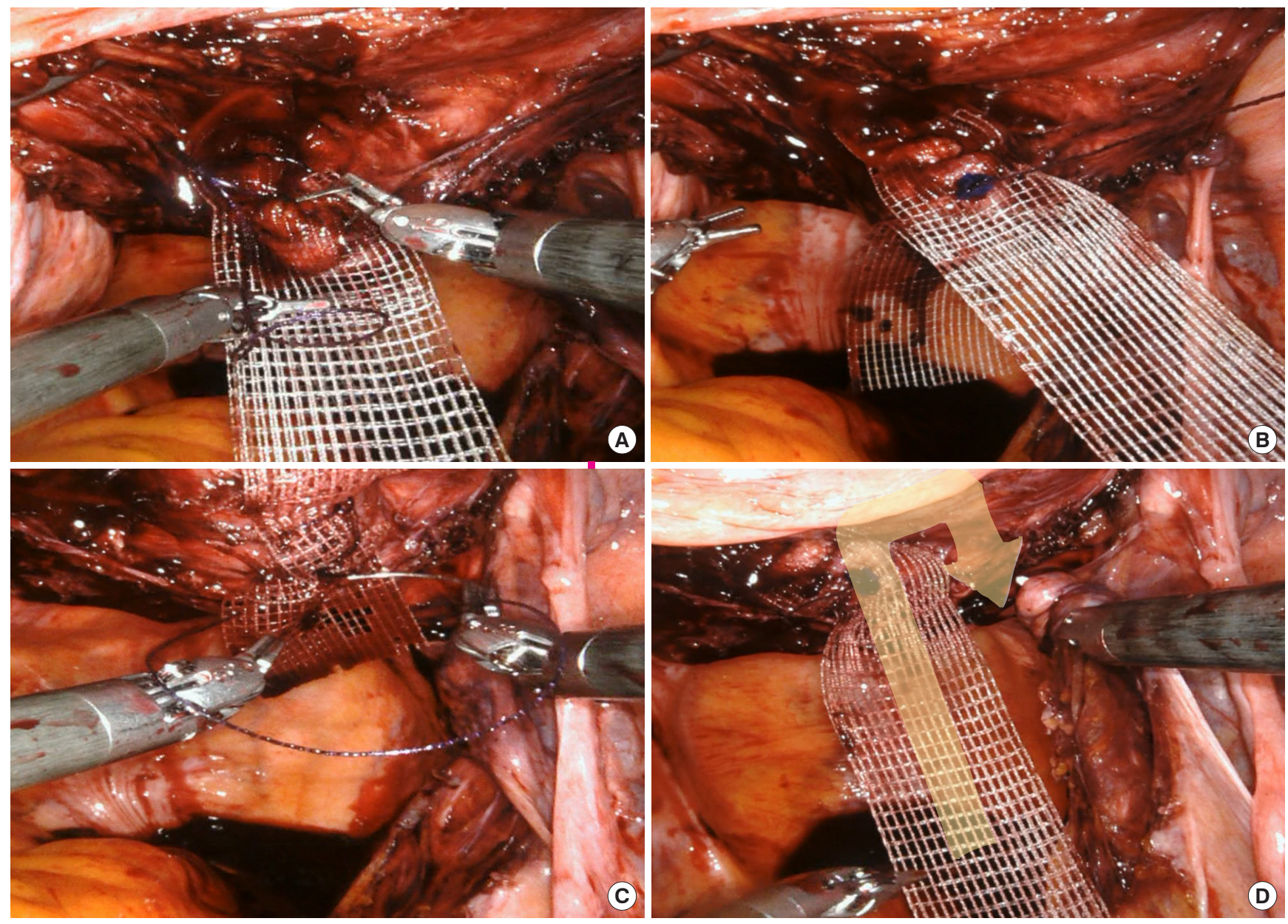

Fig. 2. Fixation of the distal mesh arms to (A, B) the anterior vaginal wall and (C) the posterior vaginal wall using a barbed, delayedabsorbable suture (V-Loc 180; Covidien, Walpole, MA, USA) in a continuous manner. (D) The proximal arm of the mesh passes through the posterior peritoneum (yellow arrow). 

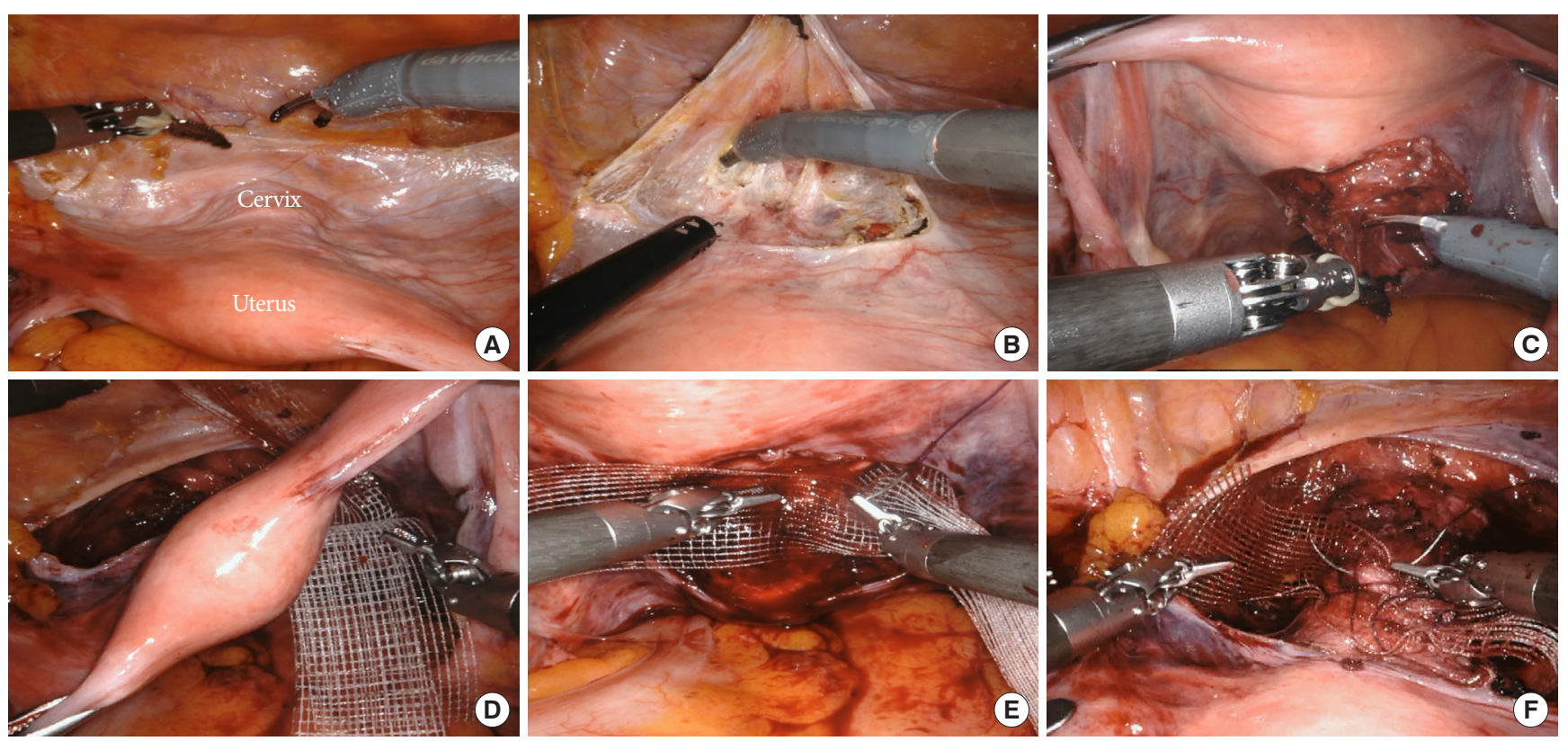

Fig. 3. Vaginal wall dissection in sacrohysteropexy (uterus-preserving). (A) Surgical anatomy of the vaginal apex and bladder. (B) Anterior vaginal wall dissection. (C) Posterior vaginal wall dissection. (D) The anterior mesh arm is tunneled through the right broad ligament. Fixation of the distal mesh arms to $(\mathrm{E})$ the posterior vaginal wall and $(\mathrm{F})$ the anterior vaginal wall using a barbed, delayedabsorbable suture (V-Loc 180; Covidien, Walpole, MA, USA) in a continuous manner.

reported to be $84 \%-100 \%$ and the subjective cure rate to be $92 \%-95 \%$ in a systematic review that analyzed studies conducted between 2006 and 2013 [8]. Studies published since then have reported similar efficacies (Table 2). However, there are major challenges when comparing research results. First, the definitions of success and failure differ among studies. Second, follow-up periods vary substantially among studies. Third, preoperative POP severity also varies among patients. Finally, a considerable amount of heterogeneity is observed at each stage of the surgical technique. Therefore, interpretation of outcomes requires caution.

In studies with an average follow-up period of 6 months to 12 months, the cure rate of apical compartment prolapse ranges from $88 \%-100 \%$ [9-15], and in those with a follow-up period of $12-24$ months, the cure rate ranges from $91.4 \%-100 \%[6,16-$ 22]. Even in long-term studies with over 5 years of follow-up, the cure rate is $93.3 \%-100 \%$, indicating that RSC has excellent durability $[23,24]$ (Table 2).

In studies conducted only on patients with advanced-stage POP, namely Baden-Walker grade 3 or Pelvic Organ Prolapse Quantification System (POP-Q) stage 3 or higher, the anatomical cure rate of apical compartment prolapse is $95 \%-100 \%$, indicating excellent outcomes [16-18,22,24-26].

\section{Concurrent Supracervical Hysterectomy or Uterine Preservation}

Because apical compartment prolapse includes not only vault prolapse that occurs in patients who have undergone prior hysterectomy, but also uterine prolapse, many studies are not limited to patients who have undergone hysterectomy. Therefore, when RSC is performed on patients with a uterus, concurrent hysterectomy or supracervical hysterectomy is performed depending on the surgeon, although hysteropexy can be performed with uterus preservation. In addition, supracervical hysterectomy and uterine preservation are performed selectively depending on the patient, even in the same study. However, in most studies, the surgical outcomes of concurrent supracervical hysterectomy or uterine preservation have not been analyzed separately. As shown in Table 1, the objective cure rate of apical compartment prolapse was reported to be $88 \%-100 \%$ in 5 studies where RSC was performed only on patients suffering from vault prolapse after hysterectomy [13,15,16,24]. Among 12 studies in which RSC was performed on patients suffering from apical compartment prolapse irrespective of prior hysterectomy (excluding 4 studies where it was unclear whether the uterus was preserved or removed during RSC), 2 studies where the uterus was always preserved reported a cure rate of $100 \%$ 

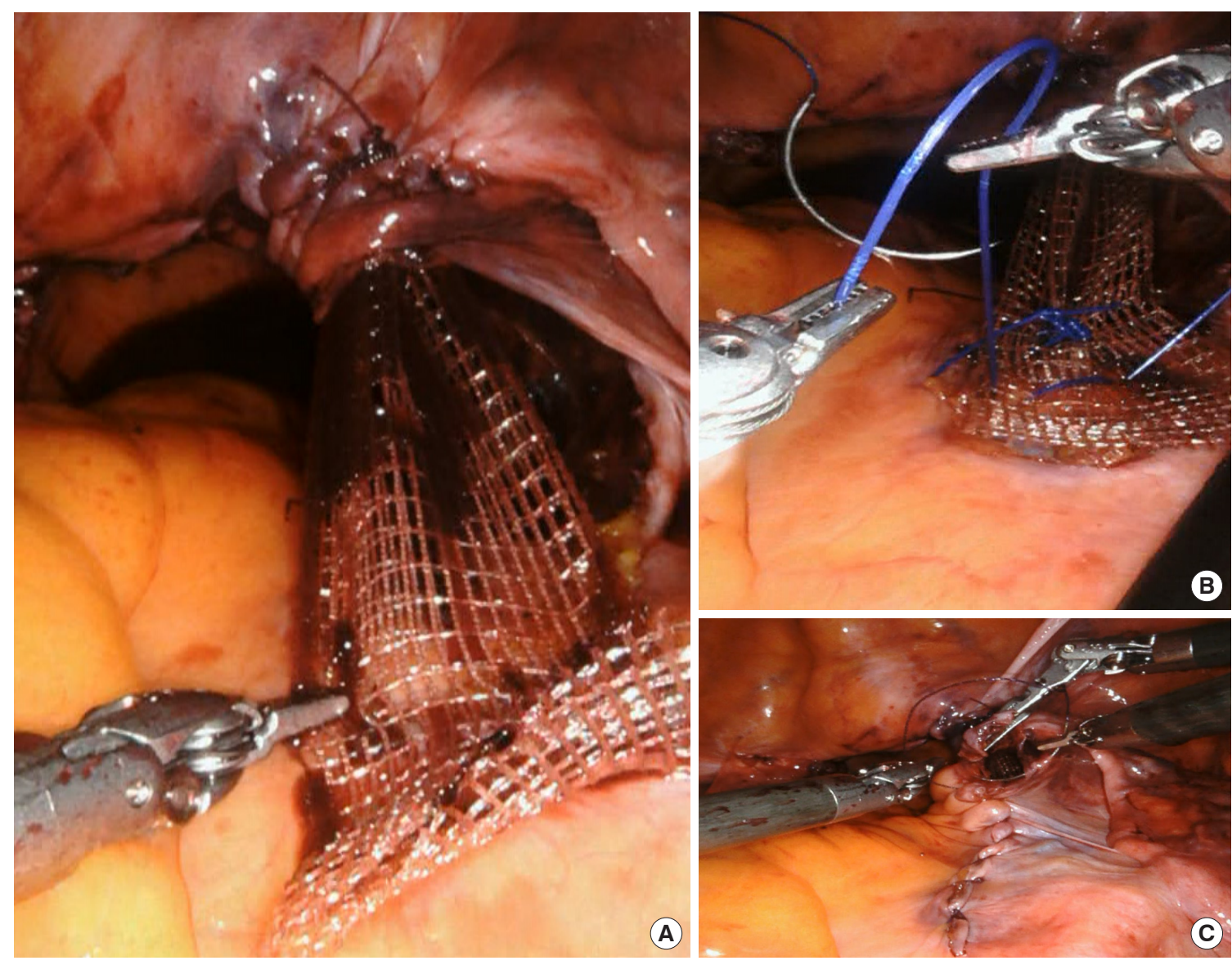

Fig. 4. Fixation of the proximal arm of the mesh. (A) At this time, the tension of the mesh should be adjusted while the vagina is restored using a vaginal manipulator. (B) The mesh is sutured to the anterior longitudinal ligament overlying the sacrum with 2-3 sutures. (C) Reperitonealization after fixation of the mesh using a barbed, delayed-absorbable suture (V-Loc 180; Covidien, Walpole, MA, USA) in a continuous manner.

[17,27], and 5 studies of concurrent supracervical hysterectomy in all patients reported cure rates ranging from $93.3 \%$ to $99.3 \%$ $[11,12,14,23,25]$. All other studies preserved the uterus or performed concurrent supracervical hysterectomy depending on the patient, with reported cure rates ranging from $94 \%$ to $100 \%$ [9,10,18,20,21,28]. Van Zanten et al. [21] performed the first prospective study of patients with symptomatic apical POP of POP-Q stage 2 or higher. In that study, the authors compared 188 patients who had undergone RSC with prior hysterectomy and 117 patients who had a uterus and had undergone robotic supracervical hysterectomy with sacrocervicopexy (RSHS). The reported cure rate of the apical compartment was $91.4 \%$ for the RSC group and 99\% for the RSHS group, while the cure rate for all compartments was $67.1 \%$ for the RSC group and $64.8 \%$ for the RSHS group. These results indicate that, in patients with a uterus, the surgical cure rate remains high. We prefer sacrohys- teropexy if there is no contraindication for uterine preservation in patients with apical prolapse [27]. Hysteropexy has advantages of maintained fertility and natural menopausal timing due to preservation of the uterus, and $36 \%-60 \%$ of female patients would choose uterine preservation assuming equal surgical efficacy [29] (Table 2).

\section{Comparative Studies}

The clinical outcomes of RSC are comparable to those of open sacrocolpopexy (Table 2). Siddiqui et al. [20] retrospectively analyzed the outcomes of RSC $(n=125)$ and open ASC $(n=322)$ in patients with POP of stage 2 or higher after prior hysterectomy. An anatomic success rate of $94 \%$ was obtained for both groups over a 12-month follow-up period. In a logistic regression analysis that controlled for parity, concomitant hysterectomy, and posterior repair, no significant differences were found 


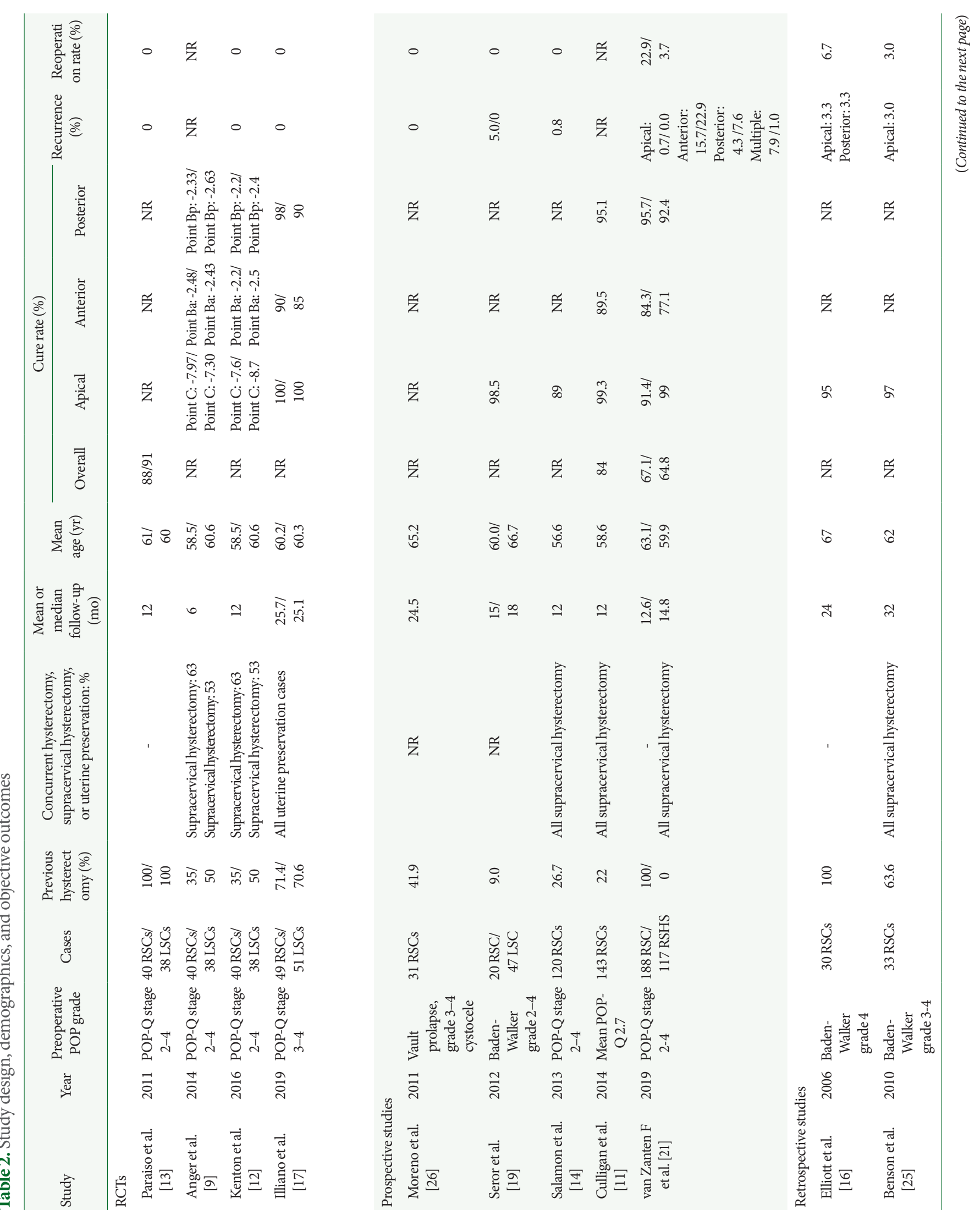




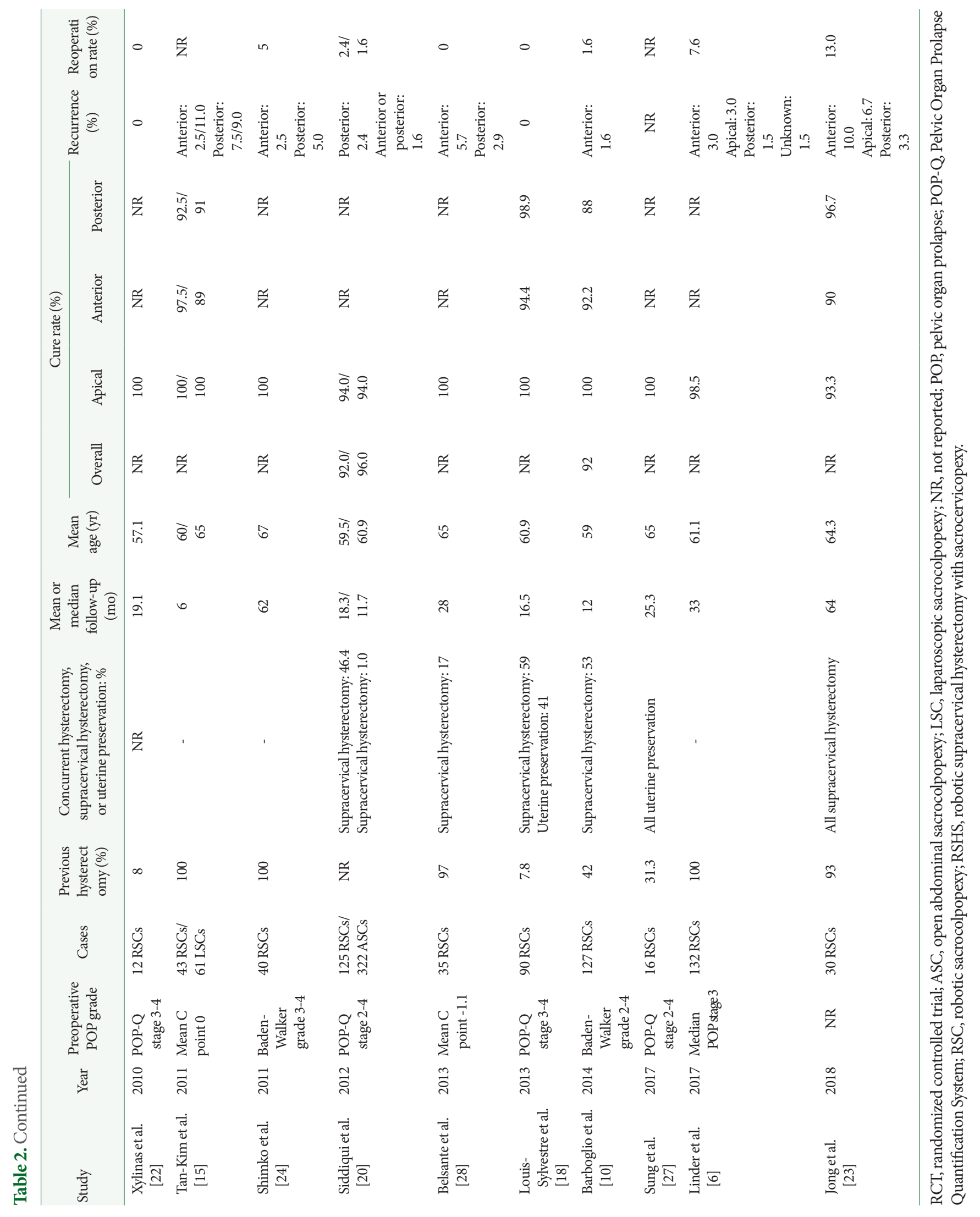


between RSC and ASC. Although the study was retrospective, the composite outcome was defined clearly; cases requiring repeat surgery due to bothersome vaginal bulge symptoms were defined as symptomatic failures, and cases of the vaginal apex descending to below the upper third of the vagina or anterior or posterior vaginal wall prolapse beyond the hymen were defined as anatomic failures. No significant differences were found in surgical failure based on composite outcomes of symptoms (RSC: 7 of 86 [8\%] vs. LSC: 12 of 304 [4\%]; P = 0.16). In addition, RSC had the advantages of reduced blood loss during surgery and reduced hospital stay, although the operative time was longer than that of open sacrocolpopexy [8].

No difference in efficacy according to surgical method has been observed, even in comparison with LSC (Table 2). In 2011, Tan-Kim et al. [15] performed a retrospective analysis of 40 patients who had undergone RSC and 61 patients who had undergone LSC and found that the cure rate of the apical compartment was $100 \%$ in both groups during an average followup period of 6 months. There have been 2 randomized controlled trials (RCTs) of LSC and RSC since then. Paraiso et al. [13] conducted a 12-month follow-up observation after RSC $(\mathrm{n}=40)$ and LSC $(\mathrm{n}=38)$ in patients with POP-Q stage 2-4 after hysterectomy. The percentage of patients who achieved POP-Q stage $0-1$ for the apical compartment was $88 \%$ in the RSC group and 91\% in the LSC group, and this difference was not statistically significant. The authors reported that the operative time for RSC was significantly longer, and that RSC was associated with more severe pain than LSC with no cost benefit. In a 2016 RCT involving patients with symptomatic apical POP of POP-Q stage 2 and above, Kenton et al. [12] revealed in that both LSC and RSC patients showed significant improvement at the $\mathrm{Ba}, \mathrm{Bp}$, and $\mathrm{C}$ points during the 12-month follow-up period compared to before surgery. Illiano et al. [17] recently reported the outcomes of an RCT of RSC $(n=49)$ and LSC $(n=51)$ in patients with POP-Q stage 3-4. When cure was defined as prolapse stage $<2$ for all compartments, point $\mathrm{C} \leq-5$, and total vaginal length of at least $7 \mathrm{~cm}$, a $100 \%$ success rate for the apical compartment was reported in both groups during an average follow-up period of 24.1 months (Table 2).

\section{Recurrence and Reoperation}

It is challenging to define the success of POP repair. Is anatomical restoration to the original state a success? Is elimination of bulging symptoms felt by the patient a success? It is important to assess composite outcomes to address this issue. The impor- tance of composite outcomes was clearly demonstrated by the CARE trial, which reported stratified outcomes as subjective, anatomic, or composite failure after POP repair. Anatomic failure after sacrocolpopexy was defined as postoperative POP requiring reoperation or pessary or recurrent prolapse according to the POP-Q system, defined as the vaginal apex descending to below the upper third of the vagina, or anterior or posterior vaginal wall prolapse beyond the hymen. Interestingly, half of the patients with anatomic failure at the 7-year follow-up reported no symptoms and did not require further treatment [30]. Culligan et al. [11] evaluated the clinical cure rate, as well as the conventional objective anatomic cure rate, in a prospective study of 143 RSC patients. Clinical cure was defined as (1) no reoperation for POP, (2) no symptoms of vaginal bulge, (3) no POP-Q point $>0$, (4) POP-Q point $C \leq-5$, and (5) an answer of "satisfied" or "very satisfied" on SSQ-8 (surgical satisfaction questionnaire 8). The clinical cure rate was $95 \%$ (136 of 143), higher than the anatomic cure rate of $84 \%$ based on the overall POP-Q stage. The authors further reported that 1 of the patients had a point $\mathrm{C}$ of -4 , corresponding to clinical failure, but no POP symptoms, and was very satisfied with the outcomes of surgery. In 2 other patients, the POP-Q stage was restored to 0 or 1 , but POP symptoms remained according to the pelvic floor distress inventory-20 questionnaire. In one of the largest prospective studies published by van Zanten et al. [21], the recurrence of apical prolapse was only $0.7 \%$ ( 1 of 140 ) for 12.6 months after RSC $(n=188)$, but the recurrence rates for anterior compartment prolapse and posterior compartment prolapse were $15.7 \%$ (22 of 140) and 4.3\% (6 of 140), respectively. However, reoperation was not required for symptomatic recurrence in $12.1 \%$ (17 of 140) of anterior compartment prolapse cases and 1.4\% (2 of 140) of posterior compartment prolapse cases. Nevertheless, the reoperation rate of $8.3 \%$ for recurrence of anterior prolapse is still much higher than the rates for other compartments. In a long-term follow-up observational study conducted on 30 patients who were available for monitoring for more than 36 months after RSC, prolapse recurrence in the apical compartment was noted in $6.7 \%$ ( 2 of 30 ), but none of the patients required an additional operation. Among $13.3 \%$ of patients who needed a reoperation, $10 \%$ underwent anterior colporrhaphy, and 3.3\% underwent posterior colporrhaphy [23] (Table 2).

Apical compartment prolapse occurs concomitantly with anterior and posterior compartment prolapse. We previously suggested that identification and correction of apical prolapse is 
critical to reduce recurrence after POP repair, and clinically significant apical prolapse is virtually always present in cases with both anterior and posterior compartment prolapse [29]. In most cases, the anterior and posterior compartments can be restored by apical repair alone. Nevertheless, recurrence of anterior or posterior compartment prolapse after sacrocolpopexy is due to the inability of the anterior and posterior vaginal walls to be dissected as far caudally as possible. Recurrence in the anterior compartment in the aforementioned RSC series is common, but the risk appears to be low compared to that of LSC. In a study of by Tan-Kim et al. [15], although there was no recurrence of apical prolapse after RSC and LSC, anterior compartment prolapse recurred in $2.5 \%$ of patients in the RSC group and $11 \%$ in the LSC group. Dissection of the vaginal wall up to the bladder neck appears to be challenging for novice surgeons performing LSC. The extended field of view and unlimited robot arm movement are important advantages for beginners with limited laparoscopic skills.

\section{Operative Time and Endeavors to Reduce It}

The reported mean or median operative time of RSC, defined as the time from incision to closure, varies widely, from a minimum of 124.2 minutes to a maximum of 288 minutes. An interesting result is that all 3 RCTs comparing RSC and LSC reported a longer operative time for RSC than for LSC $[9,13,17]$. In one prospective study, the operative time of RSC was 125 minutes (range, 90-270 minutes), significantly shorter than the 220 minutes required for LSC (range, 80-420 minutes) [19]. The surgeons who participated in the randomized trials were laparoscopic experts and appeared to have overcome the learning curve. Therefore, the operative time of LSC was shorter. However, robot-assisted laparoscopic surgery allows suturing and maneuvers requiring dexterity to be performed more quickly than does manual laparoscopy [31], and robotics can help novices overcome the learning curve rapidly [32]. Due to the nature of sacrocolpopexy, the most time-consuming procedure is suturing for mesh fixation and reperitonealization. Therefore, a novice in minimally invasive sacrocolpopexy may take less time to perform RSC than to master LSC.

When performing RSC, additional time is needed if concurrent vaginal surgery such as anterior or posterior repair and anti-incontinence surgery is required. However, except when antiincontinence surgery such as a sling is simultaneously needed, high-grade cystocele may be satisfactorily corrected by RSC alone, and the time for vaginal surgery can be reduced [33]. In patients with a uterus, uterine preservation may facilitate a shorter operative time than concurrent hysterectomy (supracervical or total) [34].

\section{Mesh Complications and Lightweight Mesh}

A systematic review of RSC studies reported mesh erosion rates ranging from $0 \%$ to $8 \%$ [8]. U.S. Food and Drug Administration recommendations and recent trends regarding use of mesh in POP repair restrict the use of transvaginal meshes [35]. Thus far, the use of mesh through a transabdominal approach does not appear to pose a problem. In a retrospective study that followed open sacrocolpopexy and RSC patients for an average of 1 year, the mesh erosion rate was $5.3 \%$ vs. $2.4 \%$, indicating higher risk in the open sacrocolpopexy group, but without statistical significance [20]. In studies comparing LSC and RSC, mesh erosion rates of $0 \%-5 \%$ have been reported for patients undergoing RSC and $0 \%-6 \%$ for those undergoing LSC, suggesting that RSC does not reduce mesh complications. Fundamentally, the use of mesh through the abdominal approach in POP repair is associated with a significantly lower risk of meshrelated complications than the transvaginal approach. However, further long-term follow-up results are need to confirm this (Table 3).

Mesh materials have also been modified to reduce complications. Type 1 polypropylene mesh is the most commonly used material, although not all type 1 polypropylene materials are the same. In addition to mesh erosion, pain and dyspareunia can also be caused by mesh. To overcome these problems, lightweight or ultra-lightweight mesh products have been launched to reduce "mesh load." A weight-based classification of mesh materials was introduced by Earle and Mark [36]: heavyweight ( $\left.>80 \mathrm{~g} / \mathrm{m}^{2}\right)$, medium-weight $\left(50-80 \mathrm{~g} / \mathrm{m}^{2}\right)$, and lightweight $\left(<35 \mathrm{~g} / \mathrm{m}^{2}\right)$. A study comparing vaginal mesh extrusion rates between lightweight mesh and heavier mesh in patients with POP showed a $46 \%$ reduction in rate of mesh exposure in those receiving lighter-weight mesh, which may be of clinical importance [37]. Salamon et al. [14] conducted the first prospective study of an ultralight mesh, Restorelle Y Smartmesh (18.69 g/ $\mathrm{m}^{2}$, Coloplast A/S, Humlebæk, Denmark), in RSC. The authors monitored 118 patients for 1 year and reported no mesh erosion or mesh-related complications. While the application of lighter mesh materials appears to be very promising, more research is needed. Other postoperative complications are summarized in Table 3. 


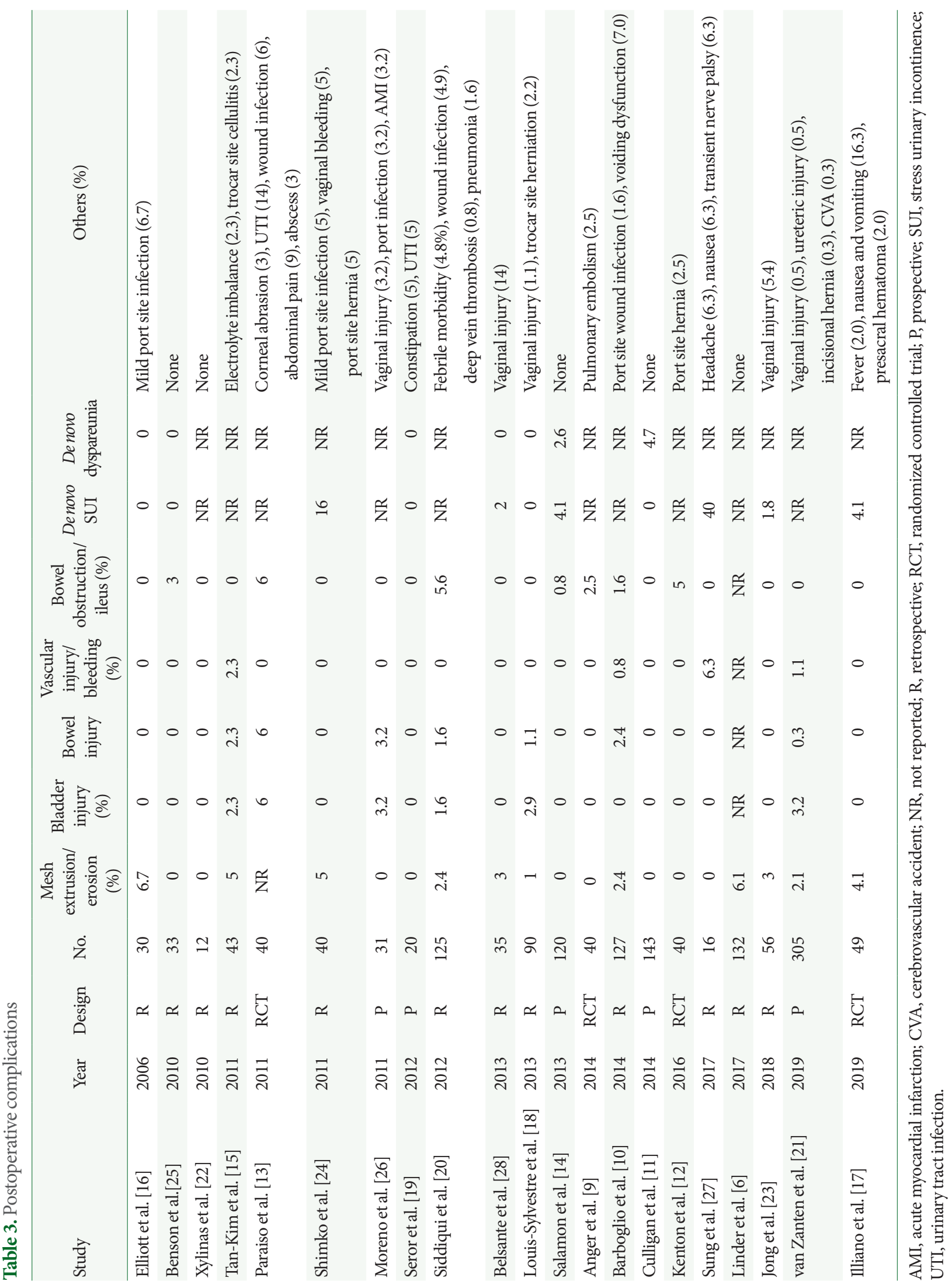




\section{CONCLUSIONS}

Sacrocolpopexy through an abdominal approach is the current gold standard for the restoration of apical compartment prolapse. RSC is of interest at this time of transition from open surgery to minimally invasive surgery because of the increased risk of morbidity associated with open surgery. Good outcomes have been achieved with RSC even in advanced-stage POP, and there are data that indicate that this technique results in durable outcomes. In terms of postoperative morbidity and complications, RSC can greatly improve patients' symptoms and quality of life. Particularly for novice surgeons who are not familiar with LSC, RSC is an excellent choice as the first surgical skill to attempt.

\section{AUTHOR CONTRIBUTION STATEMENT}

- Conceptualization: KSL

- Formal Analysis: $K J K$

- Investigation: $K J K, K S L$

- Methodology: KJK

- Project Administration: KSL

-Writing - Original Draft: KJK

- Writing - Review \& Editing: KSL

\section{SUPPLEMENTARY MATERIAL}

Supplementary video clip can be found via https://doi.org/10. 5213/inj.2040056.028 or https://www.youtube.com/watch?v= $5 \mathrm{ksQBoCUQzc} \& \mathrm{t}=13 \mathrm{~s}$.

\section{REFERENCES}

1. Wu JM, Matthews CA, Conover MM, Pate V, Jonsson Funk M. Lifetime risk of stress urinary incontinence or pelvic organ prolapse surgery. Obstetrics and gynecology 2014;123:1201-6.

2. Wu JM, Wechter ME, Geller EJ, Nguyen TV, Visco AG. Hysterectomy rates in the United States, 2003. Obstetrics and gynecology 2007;110:1091-5.

3. Wang J, Wang X, Hua K, Chen Y. Laparoscopic sacrocolpopexy plus colporrhaphy with a small intestine submucosa graft versus total pelvic floor reconstruction for advanced prolapse: a retrospective cohort study. Int Neurourol J 2019;23:144-50.

4. Ganatra AM, Rozet F, Sanchez-Salas R, Barret E, Galiano M, Cathelineau $\mathrm{X}$, et al. The current status of laparoscopic sacrocolpo- pexy: a review. Eur Urol 2009;55:1089-103.

5. Di Marco DS, Chow GK, Gettman MT, Elliott DS. Robotic-assisted laparoscopic sacrocolpopexy for treatment of vaginal vault prolapse. Urology 2004;63:373-6.

6. Linder BJ, Anand M, Klingele CJ, Trabuco EC, Gebhart JB, Occhino JA. Outcomes of robotic sacrocolpopexy using only absorbable suture for mesh fixation. Female Pelvic Med Reconstr Surg 2017; 23:13-6.

7. Tan-Kim J, Nager CW, Grimes CL, Luber KM, Lukacz ES, Brown $\mathrm{HW}$, et al. A randomized trial of vaginal mesh attachment techniques for minimally invasive sacrocolpopexy. Int Urogynecol J 2015;26:649-56.

8. Serati M, Bogani G, Sorice P, Braga A, Torella M, Salvatore S, et al. Robot-assisted sacrocolpopexy for pelvic organ prolapse: a systematic review and meta-analysis of comparative studies. Eur Urol 2014;66:303-18.

9. Anger JT, Mueller ER, Tarnay C, Smith B, Stroupe K, Rosenman A, et al. Robotic compared with laparoscopic sacrocolpopexy: a randomized controlled trial. Obstet Gynecol 2014;123:5-12.

10. Barboglio PG, Toler AJ, Triaca V. Robotic sacrocolpopexy for the management of pelvic organ prolapse: a review of midterm surgical and quality of life outcomes. Female Pelvic Med Reconstr Surg 2014;20:38-43.

11. Culligan PJ, Gurshumov E, Lewis C, Priestley JL, Komar J, Shah N, et al. Subjective and objective results 1 year after robotic sacrocolpopexy using a lightweight Y-mesh. Int Urogynecol J 2014;25:7315.

12. Kenton K, Mueller ER, Tarney C, Bresee C, Anger JT. One-year outcomes after minimally invasive sacrocolpopexy. Female Pelvic Med Reconstr Surg 2016;22:382-4.

13. Paraiso MF, Jelovsek JE, Frick A, Chen CC, Barber MD. Laparoscopic compared with robotic sacrocolpopexy for vaginal prolapse: a randomized controlled trial. Obstet Gynecol 2011;118:1005-13.

14. Salamon CG, Lewis C, Priestley J, Gurshumov E, Culligan PJ. Prospective study of an ultra-lightweight polypropylene $\mathrm{Y}$ mesh for robotic sacrocolpopexy. Int Urogynecol J 2013;24:1371-5.

15. Tan-Kim J, Menefee SA, Luber KM, Nager CW, Lukacz ES. Robotic-assisted and laparoscopic sacrocolpopexy: comparing operative times, costs and outcomes. Female Pelvic Med Reconstr Surg 2011;17:44-9.

16. Elliott DS, Krambeck AE, Chow GK. Long-term results of robotic assisted laparoscopic sacrocolpopexy for the treatment of high grade vaginal vault prolapse. The Journal of urology 2006;176:6559.

17. Illiano E, Ditonno P, Giannitsas K, De Rienzo G, Bini V, Costantini 
E. Robot-assisted Vs Laparoscopic sacrocolpopexy for high-stage pelvic organ prolapse: a prospective, randomized, single-center study. Urology 2019;134:116-23.

18. Louis-Sylvestre C, Herry M. Robotic-assisted laparoscopic sacrocolpopexy for stage III pelvic organ prolapse. Int Urogynecol J 2013;24:731-3.

19. Seror J, Yates DR, Seringe E, Vaessen C, Bitker MO, Chartier-Kastler E, et al. Prospective comparison of short-term functional outcomes obtained after pure laparoscopic and robot-assisted laparoscopic sacrocolpopexy. World J Urol 2012;30:393-8.

20. Siddiqui NY, Geller EJ, Visco AG. Symptomatic and anatomic 1 -year outcomes after robotic and abdominal sacrocolpopexy. Am J Obstet Gynecol 2012;206:435.e1-5.

21. van Zanten F, Schraffordt Koops SE, O'Sullivan OE, Lenters E, Broeders I, O'Reilly BA. Robot-assisted surgery for the management of apical prolapse: a bi-centre prospective cohort study. Bjog 2019;126: 1065-73.

22. Xylinas E, Ouzaid I, Durand X, Ploussard G, Salomon L, Gillion N, et al. Robot-assisted laparoscopic sacral colpopexy: initial experience in a high-volume laparoscopic reference center. Journal of endourology 2010;24:1985-9.

23. Jong K, Klein T, Zimmern PE. Long-term outcomes of robotic mesh sacrocolpopexy. J Robot Surg 2018;12:455-60.

24. Shimko MS, Umbreit EC, Chow GK, Elliott DS. Long-term outcomes of robotic-assisted laparoscopic sacrocolpopexy with a minimum of three years follow-up. J Robot Surg 2011;5:175-80.

25. Benson AD, Kramer BA, Wayment RO, Schwartz BF. Supracervical robotic-assisted laparoscopic sacrocolpopexy for pelvic organ prolapse. JSLS 2010;14:525-30.

26. Moreno Sierra J, Ortiz Oshiro E, Fernandez Pérez C, Galante Romo I, Corral Rosillo J, Prieto Nogal S, et al. Long-term outcomes after robotic sacrocolpopexy in pelvic organ prolapse: prospective analysis. Urol Int 2011;86:414-8.

27. Sung HH, Ko KJ, Suh YS, Ryu GH, Lee KS. Surgical outcomes and safety of robotic sacrocolpopexy in women with apical pelvic organ prolapse. Int Neurourol J 2017;21:68-74.

28. Belsante M, Murray S, Dillon B, Zimmern P. Mid term outcome of robotic mesh sacrocolpopexy. Can J Urol 2013;20:6656-61.

29. Ko KJ, Lee KS. Current surgical management of pelvic organ prolapse: Strategies for the improvement of surgical outcomes. Investig Clin Urol 2019;60:413-24.

30. Nygaard I, Brubaker L, Zyczynski HM, Cundiff G, Richter H, Gantz M, et al. Long-term outcomes following abdominal sacrocolpopexy for pelvic organ prolapse. JAMA 2013;309:2016-24.

31. Yohannes P, Rotariu P, Pinto P, Smith AD, Lee BR. Comparison of robotic versus laparoscopic skills: is there a difference in the learning curve? Urology 2002;60:39-45.

32. Chandra V, Nehra D, Parent R, Woo R, Reyes R, Hernandez-Boussard T, et al. A comparison of laparoscopic and robotic assisted suturing performance by experts and novices. Surgery 2010;147:8309.

33. Lucot JP, Cosson M, Bader G, Debodinance P, Akladios C, SaletLizee D, et al. Safety of vaginal mesh surgery versus laparoscopic mesh sacropexy for cystocele repair: results of the prosthetic pelvic floor repair randomized controlled trial. Eur Urol 2018;74:167-76.

34. Unger CA, Paraiso MF, Jelovsek JE, Barber MD, Ridgeway B. Perioperative adverse events after minimally invasive abdominal sacrocolpopexy. Am J Obstet Gynecol 2014;211:547.e1-8.

35. U.S. Food and Drug Administration. Urogynecologic Surgical Mesh: Update on the Safety and Effectiveness of Transvaginal Placement for Pelvic Organ Prolapse. Silver Spring (MD): U.S. Food and Drug Administration; 2011.

36. Earle DB, Mark LA. Prosthetic material in inguinal hernia repair: how do I choose? Surg Clin North Am 2008;88:179-201, x.

37. Moore RD, Lukban JC. Comparison of vaginal mesh extrusion rates between a lightweight type I polypropylene mesh versus heavier mesh in the treatment of pelvic organ prolapse. Int Urogynecol J 2012;23:1379-86. 